\title{
COVID 19 in pregnancy: our experience amidst second wave of COVID-19 at a teaching institute
}

\author{
Shehla Jamal*, Kainat Masroor, Akansha Tyagi, Rajini Agarwal
}

Department of Obstetrics and Gynaecology, RMRI, Uttar Pradesh, India

Received: 27 October 2021

Revised: 06 January 2022

Accepted: 07 January 2022

\author{
*Correspondence: \\ Dr. Shehla Jamal, \\ E-mail: essjayoms@gmail.com
}

Copyright: () the author(s), publisher and licensee Medip Academy. This is an open-access article distributed under the terms of the Creative Commons Attribution Non-Commercial License, which permits unrestricted non-commercial use, distribution, and reproduction in any medium, provided the original work is properly cited.

\begin{abstract}
To analyse the obstetrical outcome during second wave of coronavirus disease 2019 (COVID-19) at Level 2 facility. This is a hospital based analytical study conducted at Raj Shree medical and research institute Bareilly. During second wave a total 87 patients presenting to obstetric emergency department, over a period of one month (April 2021 to May 2021). A total of 26 patients were admitted in the hospital. 20 patients were COVID-19 positive and 6 patients were symptomatic with RT-PCR negative report. Out of 20 patients 19 were antenatal case and 1 case was postnatal. There were 2 mortalities among all positive patients. In all COVID-19 positive patients' majority of the patients were in 26 to 30 years of age (46\%). A High proportion presented in third trimester, at the gestation period between 37 to 40 weeks (54\%). Around $23 \%$ patients had severe symptoms and there were 4 ICU admissions. $65 \%$ patients required supplemental oxygen at a rate of $>12 \mathrm{l} / \mathrm{min}$. There were a total 2 mortalities; both were in severe category with rapid deterioration. The significant laboratory derangement observed was coagulation profile deranged in $40 \%$ cases. COVID-19 second wave was deleterious for obstetric population in an unprecedented manner. A high morbidity and mortality were seen in this duration. Data and evidence which is continuously evolving needs to be shared for global references and knowledge sharing amongst peers.
\end{abstract}

Keywords: COVID-19, Pregnancy, Morbidity, Mortality

\section{INTRODUCTION}

The new beta corona virus, the severe acute respiratory syndrome coronavirus-2 (SARS-COV2), known as coronavirus disease 2019 (COVID-19) grossly affected the lives of people since its onset in December 2019. With the rapid emergence of devastating second wave due to multiple mutant strains, it shook the country to a worst extent leading to desolation. Compared to the total population of India and densely populated cities like Mumbai, the death rate was also higher in the second wave and critical illness was seen at a compounded rate. Foreseemingly, it created never seen before challenges especially in obstetrics. The observed increase in numbers of pregnant women with severe COVID-19 could relate to the emergence of a more pathogenic strain of SARS-CoV2 ascertaining increased susceptibility to COVID-19 in pregnancy. ${ }^{1}$

Widespread shutdown of services lead to a major impact on obstetric population, as ANC bookings dropped by $84 \%$ and prenatal morbidities increased to $11-14 \% .^{2}$

After first wave infection rates started dropping in September, along with the number of new and active cases. Daily cases peaked mid-September with over 90,000 cases reported per-day, dropping to below 15,000 in January 2021. A second wave caused due to B.1.617 variant, which 
had down in March 2021 with first reported case in Amravati, was much larger than the first, with reporting rapid deterioration, increased morbidity and mortality in young and pregnant women, shortage of vaccines, hospital beds, oxygen cylinders and other medicines in parts of the country. By late April, India led the world in new and active cases. On 30th April 2021, it became the first country to report over 400,000 new cases in 24 hours. India had the largest number of confirmed cases in Asia. As of May 2021, India had the second-highest number of confirmed cases in the world (after the United States) with 25.4 million reported cases of COVID-19 infection and 283,248 deaths as of 19 May $2021 .^{3}$

SARS-CoV2 has affected pregnant women also to a greater extent. During initial days of COVID-19, there was huge concern about its impact on pregnant women but it did not pose a serious risk.

Reported literature from across the globe quoted a mortality rate of COVID-19 patients in first wave was at $1.41 \% .^{4}$

But in its second wave, COVID-19 has been very aggressive and its presentations are very inconsistent leading to loss of many mothers unexpectedly. ${ }^{5}$

As per our previous experience at our center from June to September 2020, there was low morbidity and mortality and cases were less severe with mild COVID symptoms, but during second wave pregnant women were seen to develop more severe symptoms of COVID-19. High morbidity with increased ICU admission and mortality was observed. There was increased incidence of requirement of supplementary oxygen support, NIV, mechanical ventilation. There was also huge a difference of opinion over timing and mode of delivery in moderate to severe disease patients. As per a report by Centre for Disease Control and Prevention, the risk of death is $70 \%$ higher in pregnant women than in non-pregnant women. At our center, COVID unit started again from 10th April 2021. So we are sharing our experience in the second wave of COVID-19 from 10 April 2021 till 22 May 2021.

\section{CASE SERIES}

In the first wave, rare controversies regarding timing and mode of the delivery were not of much concern but in second wave, this was the biggest dilemma. No definitive guidelines were available for ready referrals in early second wave. Later on, data and experience pooling by the apex obstetric facilities presented working guidelines.

At our center, we saw a higher rate of severe category admissions, higher mortality in obstetric patients as compared to first wave. Invasive interventions, higher cesareans and higher neonatal complications were observed at our center. We present our experience of twenty-six COVID obstetric patients in second wave for the purpose of experience sharing.
All the obstetric patients with RTPCR confirmed diagnosis of COVID were studied retrospectively in the department of obstetrics and gynecology, RMRI.

Table 1: Demographic profile.

\begin{tabular}{|lll|}
\hline Characteristics & $\begin{array}{l}\text { Number } \\
(\mathbf{n = 2 6})\end{array}$ & Percentage \\
\hline Total IPD patient & 26 & \\
\hline COVID positive & 20 & 77 \\
\hline COVID negative & 6 & 23 \\
\hline Age in years & & \\
\hline $18-25$ & 10 & 38 \\
\hline $26-30$ & 12 & 46 \\
\hline $31-35$ & 4 & 15 \\
\hline Parity & & \\
\hline Primi & 9 & 34 \\
\hline G2 & 12 & 46 \\
\hline G $\geq 3$ & 2 & 7 \\
\hline Post-natal & 3 & 11 \\
\hline Gestational age & & \\
\hline $6-27 / 6$ & 4 & 15 \\
\hline $28-36 / 6$ & 5 & 19 \\
\hline $37-42$ & 14 & 54 \\
\hline Post-natal & 3 & 11 \\
\hline
\end{tabular}

Table 2: COVID symptoms.

\begin{tabular}{|lll|}
\hline Particulars & $\begin{array}{l}\text { Number } \\
(\mathbf{n = 2 0})\end{array}$ & Percentage \\
\hline Severity of disease & & \\
\hline Asymptomatic & 6 & 30 \\
\hline Mild & 6 & 30 \\
\hline Moderate to severe & 8 & 40 \\
\hline Symptoms & & \\
\hline Breathlessness & 7 & 35 \\
\hline Cough & 4 & 20 \\
\hline Fever & 4 & 20 \\
\hline ARDS & 6 & 30 \\
\hline Prolonged PROM & 6 & 30 \\
\hline Severe anemia & 3 & 15 \\
\hline Scar tenderness & 2 & 10 \\
\hline GDM & 1 & 5 \\
\hline Obstructed labor & 6 & 30 \\
\hline Prolonged labor & 4 & 20 \\
\hline IUFD & 2 & 10 \\
\hline Deranged liver function & 2 & 10 \\
\hline Deranged Na ${ }^{+}, \mathrm{K}^{+}$level & 3 & 15 \\
\hline Deranged PT/INR & 12 & 60 \\
\hline Thrombocytopenia & 15 & 75 \\
\hline
\end{tabular}

We had a total of 87 obstetric patients, out of which 26 were admitted for various complains. 
We analysed patients data for demographic background, presenting complaints, obstetric parameters, and laboratory work up, mode of delivery, disease severity, ICU interventions, and rate of serious morbid complications.

Table 3: During hospitalization.

\begin{tabular}{|lll|}
\hline Particulars & $\begin{array}{l}\text { Number } \\
(\mathbf{n = 2 0})\end{array}$ & Percentage \\
\hline ICU admission & 4 & 20 \\
\hline O $_{\mathbf{2}}$ level $>$ 121/min & 12 & 65 \\
\hline NIV & 3 & 15 \\
\hline Intubation & 4 & 20 \\
\hline Vaginal delivery & 7 & 35 \\
\hline Caesarean delivery & 10 & 50 \\
\hline Abortion & 1 & 5 \\
\hline Mortality & 2 & 10 \\
\hline
\end{tabular}

In our patients, 20 had COVID-19 positive RTPCR report and 6 patients were symptomatic with RTPCR negative report. Out of 20 COVID-19 positive patients 19 were antenatal case and 1 was postnatal case. There were 2 mortalities in 20 COVID-19 positive patients and none was seen in RTPCR negative group (Table 1).

Out of the total number of patients majority affliction was seen in younger age group (46\%) and were with multiparity status with the percentage of $46 \%$. Gestational time of diagnosis was 34-41weeks (54\%) (Table 1).

The basis of complaints on admission, symptoms and findings on examination majority of the patients admit under moderate to severe category $(40 \%)$. As compared to last data presented by us, majority admissions were under asymptomatic category. ${ }^{6}$ In B.1.617 disease progression was rapid and in pregnancy the symptoms were compounded by other comorbidities like anemia also. As in $82 \%$ of severe category patients, moderate to severe anemia was also seen in our study $(15 \%)$.

Amongst all COVID positive patients, majority presented with compliant of breathlessness (35\%) and cough and fever $(20 \%)$ was also common in most of patient admitted with COVID-19. In a large multicentric study done at China, fever and cough were the commonest presentation, followed by breathlessness. ${ }^{7}$ Our observation might be different because of a small sample size, and majority of the patients were referred from level 1 hospitals.

In addition to ARDS associated with COVID infection, many patients presented with obstetric and medical complications like prolonged leaking, obstructed labour, scar tenderness and majority had anemia as an associated complication. Obstetric population suffered maximally as routine bookings were closed, movements were restricted and obstetric units were converted to covid facilities. Worldwide over, obstetric complications, morbidity and mortalities were reported in unprecedented frequencies and similarly so at our center also. ${ }^{8}$

Coagulation cascade derangements were a common pathology in COVID infection $(60 \%)$ and Heparin remained one of the cornerstones of treatment of this sinister entity. Commonly observed component dysfunction was thrombocytopenia $(75 \%)$ and elevated Ddimer levels. Similar findings were seen in our patients also, as thrombocytopenia and prolonged prothrombin time was seen in disproportionately higher proportion in our patients. Low molecular weight heparin was given in therapeutic doses at our center to all the moderately symptomatic patients. We report a high incidence of severe oligohydramnios also, higher occurrence of perinatal complications. According to one study, stillbirth rate was as high as 15.3 per 1000 patients (Table 2).

We noted a higher ICU admission rate (20\%) and similar rates $(11-24 \%)$ were observed by various other authors also. Oxygen concentration level required to maintain SPO2 was higher (>12 1/min) and mechanical ventilation was done in $20 \%$ of our patients, which is corroborating with the findings of contemporary authors from India. The study done by Mahajan et al also concluded a higher rate of ICU admissions, and a high case fatality rate of $8 \%$ (Table 3).

When we studied the mode of delivery, a higher caesarean delivery rate was observed. As there were no standard guidelines regarding the safest mode of delivery in moderate to severe symptoms category, we adhered to our own protocol which was formulated under the guidelines given by the apex institutions from the country. Seven $(35 \%)$ were delivered vaginally, ten $(50 \%)$ patients underwent caesarean section, and one woman had inevitable abortion (Table 3 ).

There were two fatalities observed at our center. First patient was full term pregnant (40 weeks) with COVID19 , with symptoms of full-blown breathlessness and saturation of $55-56 \%$. First case warranting a discussion was a second gravida with 42 weeks gestational having previous caesarean delivery was received in COVID triage. She had a saturation of 50 to $60 \%$ and had severe anemia (Hb 3 gm\%) FHS was 90 beats per minute and on internal examination thick meconium was present. Patient had already been refused admission from three other COVID facilities due to non-availability of beds while being shifted to OT theatre she started gasping and was intubated. Her perimortem caesarean was not attempted as IUFD occurred during maternal resuscitation. Non availability of admitting facilities was a major issue in the second wave and led to a greater number of complications observed.

Second case is discussed in view of gradual deterioration. Patient was primi with 20 weeks 4 days duration. She conceived after infertility treatment and was on progesterone support on the day of her admission platelet 
was 60,000 and the D dimer levels were very high 1.5 . She developed bleeding through vagina and landed into incomplete abortion. The process was hastened using medical therapy, her platelets showed a decreasing trend as well her saturation deteriorated. She was intubated and succumbed within 4 hours, some authors have reported an increased incidence of coagulation abnormalities in females taking hormonal contraception, few observations reflect association of progesterone and coagulopathies. Nonetheless, the second wave of the pandemic brought with its fury of nature along with reflected helplessness in the form of lesser knowledge about management with different co morbid conditions.

\section{DISCUSSION}

Our study demonstrates a higher frequency of spread, intensive care unit, high dependency unit admission and maternal deaths during the second wave of COVID. As we notice from our data presented previously and experiences from second wave differs in severity of the disease and rate of mortality in every trimester and rapid spread of disease.

It is possible that higher cases rate and delay in seeking health care are contributors to increased mortality during the second wave of the COVID-19 pandemic. Increased frequency of obstetric complications like rupture uterus and obstructed labor was seen in the patient admitted to facility because of lack of regular check-up, follow-up were not possible due to increasing cases very rapidly and patients were afraid to visit hospital frequently.

In summary the spread of infection was very rapid as comparison to the first wave even unexpectedly in such a short time infection involves great percentage of population. Severity of infection also very high in second wave, lung involvement was very quick after getting infection this leads to patients land up in to ARDS. ${ }^{9}$

In this study, COVID-19 infection during pregnancy was associated with substantial risk of morbidity and mortality, compared with their not-infected pregnant women, especially if these individuals were symptomatic. In spite of all arrangements and hard efforts there were shortage of beds in hospitals and lack of oxygen availability just because of rapid spread of infection with high severity, more people need getting admission and to maintain saturation needs oxygen.

Even arrangements were done by government as well as at every level just to avoid morbidity and mortality but all efforts failed and there were shortage of beds and oxygen. Many people die because of not availability of oxygen at right time. Many people died in between because of referral from various places due to not availability of beds in those hospitals.

\section{CONCLUSION}

COVID-19 affects people of all age groups. It neither spares people with comorbidities nor those without any comorbidities. Hence CAB (COVID appropriate behaviour) will be long lasting to minimize spread of this disease. Prevention is always better than getting affected with the disease. Now, although vaccine is available, the virus is also coming up with newer mutations. Hence, we have to be careful about this global pandemic which comes up with some unique surprises every single day. It is wisely said that Prevention is always better than getting affected with the infection. Now, even though vaccine is available, the virus is also coming up with different variations. Hence, we have to be cautious about this universal pandemic which comes up with some exceptional revelations every single day.

\section{Funding: No funding sources \\ Conflict of interest: None declared \\ Ethical approval: Not required}

\section{REFERENCES}

1. COVID-19 India. Available at: https://www.mohfw.gov.in. Accessed on 20 August 2021.

2. Charlson ME, Pompei P, Ales KL, MacKenzie CR. A new method of classifying prognostic comorbidity in longitudinal studies: development and validation. J Chronic Dis. 1987;40(5):373-83.

3. Wu Y, Zhang C, Liu H. Perinatal depressive and anxiety symptoms of pregnant women during the coronavirus disease 2019 outbreak in China. Am J Obstet Gynecol. 2020;223.

4. Davenport MH, Meyer S, Meah VL, Strynadka MC, Khurana R. Moms are not OK: COVID-19 and maternal mental health. Front Glob Womens Health. 2020;2:21-4.

5. Yap M, Debenham L, Kew T. PregCOV-19 Consortium. Clinical manifestations, prevalence, risk factors, outcomes, transmission, diagnosis and treatment of COVID-19 in pregnancy and postpartum: a living systematic review protocol. BMJ Open. 2020;10.

6. Jamal S, Singh N. COVID-19 in Pregnancy: An Experience at a Dedicated Tertiary Care COVID Facility in Western Uttar Pradesh. Journal of South Asian Federation of Obstetrics and Gynaecology. 2021;13:81-3.

7. Pandya M, Patel M, Bhesaniya J, Patel S, Patel S, Patel K et al. Pregnancy \& Covid 19 infection: Our experience. Indian Journal of Obstetrics and Gynecology Research. 2021;8:15-9.

8. Bulletin de l'Académie Nationale de Médecine. COVID-19: Interpretation of morbidity and mortality data. 2020;204:e95-6.

9. Kadiwar S, Smith JJ, Ledot S, Johnson M, Bianchi P, Singh N, et al. Were pregnant women more affected 
by COVID-19 in the second wave of the pandemic? Lancet. 2021;397:1539-40.
Cite this article as: Jamal S, Masroor K, Tyagi A, Agarwal R. COVID 19 in pregnancy: our experience amidst second wave of COVID-19 at a teaching institute. Int J Reprod Contracept Obstet Gynecol 2022;11:599-603. 